COMMENT. Glutaric acidemia type 1 (GA-1) is an autosomal recessive disorder of amino acid metabolism caused by a deficiency of glutaryl-CoA dehydrogenase, and leading to accumulation of glutaric acid, 3-OH-GA and glutaconic acid in blood, urine, CSF and brain tissue. Characteristic clinical features include macrocephaly at, or soon after, birth, an abrupt onset of dystonia between 6 and 18 months of age, often with a febrile illness, and death in early childhood. Caudate and putamen atrophy with neuronal loss are always present, and white matter spongiform degeneration is a frequent finding.

Strauss KA, in a Commentary (Brain 2005;128:697-699), stresses the clinician's dilemma in predicting when basal ganglia injury will occur (the progress of the disease can be variable), and the lack of effective therapy once the encephalopathy ensues. Plasma and urine organic acid measurements are unreliable in predicting the time of onset of neurologic symptoms. The Canadian report confirms previous findings that brain GA is very high in these patients, exceeding plasma and CSF levels two-fold, an accumulation caused either by a selective blood-brain-barrier affinily for GA or by an excess brain tissue production of GA, arising from the enzymatic block in degradation of lysine and tryptophan in the brain. The risk of brain injury in GA-1 may be inversely related to the efficiency of brain organic acid clearance. The commentary emphasizes the need to consider inter-organ transport mechanisms for GA and the prevention of striatal necrosis.

\title{
POLGI MUTATIONS IN INFANTILE HEPATOCEREBRAL SYNDROMES
}

Nine patients, 2 sibling pairs and 5 singleton cases, with POLGI mutations associated with infantile fatal encephalopathy and hepatopathy, 8 having typical Alpers' syndrome (Alpers' hepatopathic poliodystrophy) and one a severe floppy infant syndrome with hepatic failure, are reported from the National Institute of Neurology, Milano; Meyer Children's Hospital, Florence; University of Verona; University Hospital, Monza, Italy; and University Children's Hospital, Hamburg, Germany. Patient 1, a boy, developed a torticollis after a few months of life and 2 episodes of sudden head drop at 10 months, followed by psychomotor arrest and regression, hypotonia, ataxia and myoclonus, first focal and later, generalized, with weakness requiring ventilatory assistance. Seizures were accompanied by a disorganized basal EEG pattern and multiple foci of paroxysmal activity. Brain MRI showed symmetrical lesions of basal ganglia, thalami, cerebellar dentate nuclei, and left occipital cortical and subcortical regions. MRS revealed an abnormal accumulation of lactic acid in the putamen and a reduction of the $\mathrm{N}$-acetyl aspartate peak, an index of neuronal loss. Cholestatic jaundice, hypoglycemia and hypocoagulation followed and were accompanied by neurologic deterioration and death at 30 months of age. In two of the 8 patients with Alpers' syndrome, severe acute liver failure followed administration of valproate for control of myoclonus. Autopsy performed in 3 cases of Alpers' syndrome showed diffuse encephalomalacia and severe liver steatosis with lobular fibrosis and bile ductile proliferation. Analysis of POLGI, a major disease gene in mitochondrial disorders, revealed that all patients carried different allelic mutations, 2 nonsense and 7 missense changes, associated with a heterogeneous spectrum of clinical outcomes. (Ferrari G, Lamantea E, Donati A et al. Infantile hepatocerebral syndromes associated with mutations in the mitochondrial DNA polymerase-

gA. Brain April 2005;128:723-731). (Respond: Massimo Zeviani MD PhD, Unit of 
Molecular Neurogenetics, National Neurological Institute Carlo Besta, via Temolo 4, 20126 Milan, Italy).

COMMENT. The clinical syndromes associated with POLGI mutations, the gene encoding the subunit of mitochondrial DNA (mtDNA) polymerase (pol-yA), include familial progressive external ophthalmoplegia, autosomal recessive sensory atactic neuropathy with ophthalmoplegia, juvenile sensory and cerebellar atactic syndrome with myoclonus epilepsy, and Alpers' hepatopathic poliodystrophy, first described by Alpers in 1931, and later by Ford (1951), Blackwood et al, 1963, and Huttenlocher et al, 1976. Huttenlocher emphasized the coincident hepatic cirrhosis. Alpers' syndrome is characterized by refractory seizures (epilepsia partialis continua), progressive neurologic deterioration, and progressive hepatic failure. The present report shows that POLGI, found in 8 of 10 cases examined over 10 years, is a prevalent disease gene in Alpers' syndrome, and tissue-specific, partial mtDNAdepletion is a molecular feature of the disease.

The hepatic complication has sometimes been attributed to valproate toxıcity during treatment of refractory seizures, and 2 of the above Italian cases received valproate just prior to acute liver failure. In a report of 13 cases of Alpers' syndrome (termed progressive neuronal degeneration) from Great Ormond Street Hospital, London, UK (Egger $\mathrm{J}$ et al. Clinical Pediatrics 1987;26:167-173), 4 patients received sodium valproate and 2 of the 4 died. Both had abnormal liver enzymes before treatment, and valproate was not accepted as the primary cause, a genetically determined metabolic explanation being preferred.

\section{DEVELOPMENTAL DISORDERS}

\section{IMPROVED GLOBAL AND LOCOMOTOR DEVELOPMENT FOLOWING SURGERY FOR SAGITTAL SYNOSTOSIS}

Twenty eight children with sagittal synostosis (SS) were assessed pre- and postoperatively and their psychomotor development was compared with that in 28 normal controls and with 13 children with SS without surgical intervention, in a prospective longitudinal design study at St James's University Hospital, Leeds, UK. Using the Griffiths Mental Developmental Scales, children with SS had significantly lower gross locomotor function scores than normal controls, $35.7 \%$ having LD scores, when tested at a mean age of 8 months. Following surgical intervention (at a mean age of 6.9 months), the global development improved $(\mathrm{p}=0.001)$, locomotor deficit resolved $(\mathrm{p}=0.0001)$, often suddenly, particularly the delay in head control, and the developmental attainment continued to improve over time (11 children had a second postoperative assessment and 4 had a third). A lesser improvement was demonstrated in eye-hand coordination and performance skills $(\mathrm{p}=0.05)$. Children with SS not surgically corrected failed to show the improvement in development demonstrated in operated cases. Surgery for SS is more than a cosmetic procedure, but the mechanism for the improved development is not definitely determined. (Bellew M, Chumas P, Mueller T et al. Pre- and postoperative developmental attainment in sagittal synostosis. Arch Dis Child April 2005;90:346-350). (Respond: Dr M Bellow, Department of Plastic, Reconstructive, and Hand Surgery, St James's University Hospital, Beckett Street, Leeds LS9 7TF, UK). 